Original Paper http://ajol.info/index.php/ijbcs http://indexmedicus.afro.who.int

\title{
Evolution des paramètres biochimiques chez les chevaux de sport pendant un test d'effort
}

\author{
A. SOW $^{1 *}$, A. BATHILY ${ }^{1}$, M. KALANDI ${ }^{1}$, R.S. TINE ${ }^{2}$ et G.J. SAWADOGO ${ }^{1}$ \\ ${ }^{I}$ Ecole Inter-Etats des Sciences et Médecine Vétérinaires (EISMV), Laboratoire d'Endocrinologie et de Radio- \\ Immunologie, BP. 5077, Dakar Fann, Sénégal. \\ ${ }^{2}$ Service Vétérinaire de la Gendarmerie Nationale, Caserne Samba Diéry Diallo, \\ BP. 4011 Dakar Colobane, Sénégal. \\ *Auteur correspondant; Tél. (+221) 3386510 08, Fax. (+221) 338254283 , \\ E-mail: wosamada@yahoo.fr ; a.sow@eismv.org
}

\section{RESUME}

L'étude avait pour objectif d'évaluer les modifications des paramètres biochimiques chez les chevaux de sport (course de saut d'obstacle) durant un test d'effort. Vingt-trois chevaux de sport de race barbe espagnol âgés de 9,34 $\pm 1,02$ ans ont servi d'animaux d'expérimentation. Des prélèvements sanguins ont été effectués sur chaque animal avant, juste après et 30 minutes après le test d'effort. Les variations significatives des paramètres biochimiques ont été notées. Les valeurs de l'hématocrite, de la créatinine kinase (CK), de l'aspartate aminotransférase (ASAT), de la créatinine, des protéines totales et du phosphore ont varié significativement selon la période de prélèvement $(\mathrm{p}<0,05)$. Le niveau de compétition a eu aussi un effet significatif sur les valeurs des protéines totales, de l'albumine, du phosphore, du calcium, du magnésium et du glucose $(\mathrm{p}<0,05)$. De même, les valeurs de l'ASAT, de la créatinine, de l'albumine et du glucose $(\mathrm{p}<0,05)$ ont varié significativement en fonction de la fréquence de compétition. L'évaluation de la forme de ces chevaux de sport est d'une grande utilité dans l'appréciation des conditions physiques.

() 2016 International Formulae Group. All rights reserved.

Mots clés : Cheval de sport, paramètres biochimiques, test d'effort, Sénégal.

\section{Evolution of biochemical parameters in sport horses subjected to a stress test}

\begin{abstract}
The study aimed to assess changes in biochemical parameters in jumping race horses during a stress test. Twenty-three Spanish barb racehorses aged in average of $9.34 \pm 1.02$ years were used as experimental animals. Blood samples were collected from each animal before, immediately after and 30 minutes after the stress test. Significant changes in biochemical parameters were noted. The values of hematocrit, Creatinine Kinase, Aspartate aminotransferase (AST), creatinine, total proteins and phosphorus varied significantly according to the sampling period $(\mathrm{p}<0.05)$. The level of competition had also a significant effect on the values of total proteins, albumin, phosphorus, calcium, magnesium and glucose $(p<0.05)$. Similarly, the values of AST, creatinine, albumin and glucose $(\mathrm{p}<0.05)$ varied significantly depending on the competition frequency. The evaluation of the fitness of these racehorses is of great use in assessing the physical conditions.

(C) 2016 International Formulae Group. All rights reserved.
\end{abstract}

Keywords: Racehorses, biochemical parameters, stress test, Senegal 


\section{INTRODUCTION}

Le cheval est une espèce sélectionnée depuis de longue date pour sa capacité à fournir des efforts physiques variés. Le cheval est sélectionné pour ses aptitudes sportives exploitées dans les courses et les sports équestres. L'essor considérable qu'ont connu ces dernières années les connaissances en matière de physiologie de l'effort chez les équidés en est l'incontestable preuve (Art et al., 2000). De nos jours, il est donc possible d'envisager le suivi de l'entrânement par la réalisation d'épreuves d'effort standardisées permettant d'estimer la capacité de l'organisme à répondre à certains types d'exercices physiques. En effet, le test d'effort consiste à faire subir au cheval, quelques jours avant l'épreuve, un test qui reproduit aussi fidèlement que possible les conditions de la course à venir. De nombreux travaux ont été déjà consacrés à l'évolution des paramètres biochimiques du cheval de course à l'effort, soit dans le but d'améliorer les techniques d'entraînement, soit le plus souvent dans l'espoir de mettre au point un test qui permette d'évaluer les chances que présente l'animal de gagner l'épreuve dans laquelle il est engagé (Çötelioğlu et al., 2001 ; Sribhen et al., 2007 ; Kedzierskiet al., 2009).

L'objectif de cette étude est d'évaluer les modifications des paramètres biochimiques des chevaux de sport avant et après l'effort de saut d'obstacle en fonction du niveau et la fréquence des compétitions.

\section{MATÉRIEL ET MÉTHODES}

\section{Chevaux sélectionnés}

L'étude a été menée à l'Escadron Monté de la Gendarmerie Nationale, situé dans l'enceinte de la Caserne Samba Diéry Diallo sise au quartier Médina à Dakar. Les chevaux de l'étude recevaient quotidiennement une ration alimentaire composée de fanes d'arachide (6kg/jour/cheval), de concentrés industriels ( $3 \mathrm{~kg} / \mathrm{jour} / \mathrm{cheval})$, du mil $(2 \mathrm{~kg} / \mathrm{jour} / \mathrm{cheval})$ et des compléments minéraux (une poignée de sel). L'étude a porté sur 23 chevaux barbes espagnols âgés de 9,34 $\pm 1,02$ ans, dont 3 hongres et 20 mâles entiers. Les données portant sur le niveau de compétition ont été rélevées pour chaque cheval. Tous les sujets de cette étude étaient des chevaux de compétition de saut d'obstacle et apparemment en bonne santé (après un examen clinique). Les juments et les sujets très jeunes ont été exclus de l'étude.

Les sujets de l'étude étaient répartis en trois catégories selon le niveau de compétition (haut, moyen et débutant) et en deux lots selon la fréquence de compétition à savoir les chevaux qui compétissent régulièrement et ceux qui n'ont pas encore effectué de sortie officielle. Les chevaux étaient soumis au test d'effort suivant: (5 mn de marche, $10 \mathrm{mn}$ de trot, $5 \mathrm{mn}$ de galop, parcours de 6 obstacles). La hauteur des obstacles varie en fonction des niveaux de compétition: (1m) pour les chevaux de haut niveau; $(90 \mathrm{~cm})$ pour les chevaux de niveau moyen et $(70$ à $80 \mathrm{~cm})$ pour les chevaux de niveau débutant.

\section{Détermination des paramètres biométriques \\ Pour chaque cheval, des mesures} barymétriques telles que la hauteur au garrot (HG), la longueur du tronc (LT) et le périmètre thoracique (PT) ont été mesurées comme décrits par Bathily et al. (2014). Brièvement, la HG a été mesurée à l'aide d'une toise à niveau au point le plus haut du garrot, le cheval étant sur une surface plane et avec une hauteur de tête normale. La LT a été évaluée avec un ruban-mètre de la pointe de l'épaule (processus de l'olécrane) à la pointe de la fesse (pointe de l'ischium). Le PT a été également déterminé à l'aide d'un rubanmètre au niveau du passage de sangle après l'expiration.

Le poids vif $(\mathrm{PV})$ en $\mathrm{kg}$ a été calculé à partir du tour de poitrine et de la hauteur au garrot d'après les formules proposées par l'Institut Nationalde la Recherche Agronomique de France selon la formule développée par Martin-Rosset (1990) :

$\mathbf{P V}=4,3 \mathrm{TP}+3 \mathrm{HG}-\mathbf{7 8 5}$

PV (kg), TP (cm), HG (cm). 


$\begin{aligned} & \text { Détermination des paramètres } \\ & \text { biochimiques } \\ & \text { Des prélèvements sanguins par }\end{aligned}$
ponction de la veine jugulaire dans des tubes secs et EDTA stériles (Vacutainer ${ }^{\circledR}$ ), ont été réalisés sur chaque animal: avant le test d'effort $\left(t_{1}\right)$, immédiatement après le test d'effort au bord de la piste $\left(\mathrm{t}_{2}\right)$, puis après un repos de $30 \mathrm{mn}\left(\mathrm{t}_{3}\right)$. Le sang prélevé sur tube sec a été centrifugé (3500 tours/mn, $15 \mathrm{mn}$ ) et le sérum recueilli a été conservé à $-20^{\circ} \mathrm{C}$. Le sang collecté sur tube EDTA (Ethyléne Diamine Tétra-Acétique) a été utilisé pour la mesure de l'hématocrite. Les analyses biochimiques ont concerné les métabolites (les protéines totales, l'albumine, la créatinine et l'urée), l'aspartate amino-transférase (ASAT), la créatine kinase $(\mathrm{CK})$, la lactate déshydrogénase (LDH); et les ions (le magnésium, le phosphore et le calcium).

Les paramétres biochimiques ont été évalués sur tous les animaux avant le test d'effort $\left(t_{1}\right)$, juste après $\left(t_{2}\right)$ et $30 \mathrm{mn}$ après $\left(t_{3}\right)$ par dosage colorimétrique à l'aide de kits commerciaux BIOSYSTEMS ${ }^{\circledR}$, S.A (Barcelona, Spain).

L'électrophorèse en zone sur gel d'agarose a été réalisée sur chaque échantillon en utilisant le kit d'électrophorése Hydragel protéine K20 SEBIA $^{\circledR}$ (France). Le profil électrophorétique représente les différentes fractions: albumine, alpha1-globuline, alpha2-globuline, bêta globuline et gamma globuline. La concentration absolue de chaque fraction est obtenue automatiquement par le densitomètre par multiplication de la concentration en protéines totales de l'échantillon par le pourcentage correspondant à la fraction.

\section{Analyses statistiques}

Les données ont été analysées avec le logiciel STATA SE9.2 ${ }^{\circledR}$. Pour chaque paramètre, la moyenne et l'écart type ont été calculés. L'analyse de variance ANOVA et le t-test ont été utilisés pour comparer les écarts entre les valeurs moyennes des paramètres obtenues des différentes périodes $\left(\mathrm{t}_{1}, \mathrm{t}_{2}\right.$ et $\left.\mathrm{t}_{3}\right)$ en fonction du niveau et de la fréquence de compétitions. Les différences ont été considérées statistiquement significatives si $\mathrm{p}<0,05$.

\section{RÉSULTATS}

\section{Paramètres morphobiométriques de la population d'étude}

Les moyennes des paramètres biométriques de la population totale étaient de $157,13 \pm 3,5 \mathrm{~cm}, 179,73 \pm 5,1 \mathrm{~cm}, 166,43 \pm 6,7$ $\mathrm{cm}$ respectivement pour la hauteur au garrot (HG), le périmètre thoracique (PT) et la longueur du tronc (LT). Le poids vif moyen estimé était de $459,26 \pm 28,8 \mathrm{~kg}$ pour l'ensemble des chevaux échantillonnés.

\section{Variations des paramètres biochimiques selon la période de prélèvement}

La période de prélèvement a eu un effet significatif sur les valeurs moyennes de l'hématocrite $(\mathrm{p}=0,000)$, de la CK $(\mathrm{p}=0,045)$, de l'ASAT $(\mathrm{p}=0,046)$, de la créatinine $(\mathrm{p}=0,000)$, des protéines totales $(\mathrm{p}=0,002)$ et du phosphore $(\mathrm{p}=0,005)$ (Tableau 1). Les valeurs moyennes de l'hématocrite, de la CK, de l'ASAT, de la créatinine, des protéines totales et du phosphore des chevaux à la (période $\mathrm{t}_{2}$ ) étaient supérieures à celles des deux autres périodes $\left(t_{1}\right.$ et $t_{3)}$. Toutefois, il n'y a pas eu de variation significative entre les valeurs moyennes de l'hématocrite, de l'ASAT, des protéines totales et du phosphore entre les périodes $t_{1}$ et $t_{3}(p>0,05)$.

\section{Variations des paramètres biochimiques selon le niveau de compétition}

Les Tableaux 2, 3 et 4 montrent les moyennes des paramètres biochimiques en fonction du niveau de compétition. Le niveau de compétition a eu un effet significatif sur les valeurs moyennes des protéines totales, de l'albumine, du phosphore, du calcium, du magnésium et du glucose $(p<0,05)$. Les 
moyennes du calcium et magnésium obtenues aux périodes $t_{2}$ et $t_{3}$ chez les chevaux de haut niveau de compétition étaient significativement plus basses que celles des chevaux des deux autres niveaux (moyen et débutant). De même, les moyennes des protéines totales (périodes $\mathrm{t}_{3}$ ) et de l'albumine (périodes $t_{2}$ ) étaient significativement plus basses chez les chevaux de haut niveau. Par contre, les moyennes du phosphore (périodes $\mathrm{t}_{2}$ ) et $\mathrm{du}$ glucose (périodest $\mathrm{t}_{2}$ et $\mathrm{t}_{3}$ ) étaient significativement plus élevées chez ces derniers.

\section{Variations des paramètres biochimiques selon la fréquence de compétition}

La fréquence de compétition a eu un effet significatif sur les valeurs moyennes de
l'ASAT, de la créatinine, de l'albumine et du glucose $(p<0,05)$ (Tableaux 5 et 6$)$. Néanmoins, certaines variations de ces paramètres aux périodes $t_{2}$ et $t_{3}$ ont été notées selon que les chevaux étaient en compétition régulièrement ou n'avaient pas encore effectué de compétition officielle. Les valeurs moyennes de l'ASAT (période $\mathrm{t}_{3}$ ), de la créatinine (période $t_{2}$ ), de l'albumine (période $t_{2}$ ) et du glucose (période $t_{3}$ ) des chevaux qui compétissaient régulièrement étaient significativement inférieures à celles des chevaux qui n'avaient pas encore effectué de compétition officielle pour les mêmes périodes.

Tableau 1 : Moyennes des paramètres biochimiques en fonction des périodes de prélèvement.

\begin{tabular}{|c|c|c|c|c|c|}
\hline $\begin{array}{l}\text { Paramètres } \\
\text { Biochimiques }\end{array}$ & $\begin{array}{l}\text { Période } t_{1} \\
(n=23)\end{array}$ & $\begin{array}{l}\text { Période } t_{2} \\
(n=23)\end{array}$ & $\begin{array}{c}\text { Période } t_{3} \\
(n=23)\end{array}$ & $\mathbf{F}$ & p-value \\
\hline Hématocrite (\%) & $35,39 \pm 3,1$ & $49,30 \pm 3$ & $37,08 \pm 1,9$ & 169,5 & $0,000^{* *}$ \\
\hline CK (UI/l) & $176,1 \pm 41,9$ & $215,95 \pm 66,3$ & $188,26 \pm 52,4$ & 3,23 & $0,045^{*}$ \\
\hline ASAT (UI/l) & $222,1 \pm 38,5$ & $249,95 \pm 40,6$ & $225,47 \pm 42,6$ & 3,21 & $0,046^{*}$ \\
\hline LDH (UI/l) & $414,9 \pm 143,8$ & $469,7 \pm 132,6$ & $478 \pm 148,4$ & 1,34 & 0,268 \\
\hline Urée (mmol/l) & $5,98 \pm 1$ & $5,99 \pm 0,9$ & $6,51 \pm 0,9$ & 2,16 & 0,123 \\
\hline Créatinine $(\mu \mathrm{mol} / \mathrm{l})$ & $136,69 \pm 12,7$ & $157,91 \pm 18,5$ & $153,52 \pm 16$ & 11,36 & $0,000^{* *}$ \\
\hline $\begin{array}{l}\text { Protéines totales } \\
(\mathrm{g} / \mathrm{l})\end{array}$ & $60,5 \pm 6$ & $66,58 \pm 6,8$ & $60,54 \pm 6,5$ & 6,65 & $0,002^{*}$ \\
\hline Albumine (g/l) & $26,9 \pm 3$ & $28,94 \pm 2,7$ & $26,95 \pm 3,6$ & 3,08 & 0,052 \\
\hline Globulines $\alpha 1(\mathrm{~g} / \mathrm{l})$ & $2,91 \pm 0,4$ & $3,2 \pm 0,6$ & $2,83 \pm 0,5$ & 1,33 & 0,280 \\
\hline Globulines $\alpha 2(\mathrm{~g} / \mathrm{l})$ & $7,21 \pm 1$ & $7,21 \pm 1$ & $6,47 \pm 0,8$ & 1,81 & 0,182 \\
\hline Globulines $\beta$ (g/l) & $7,2 \pm 1,5$ & $7,62 \pm 1,8$ & $7 \pm 1,4$ & 0,40 & 0,675 \\
\hline Globulines $\gamma(\mathrm{g} / \mathrm{l})$ & $17,13 \pm 2,8$ & $18,01 \pm 4$ & $16,7 \pm 3,2$ & 0,38 & 0,684 \\
\hline Magnésium (mg/dl) & $2,58 \pm 0,5$ & $2,65 \pm 0,5$ & $2,44 \pm 0,6$ & 0,87 & 0,425 \\
\hline Calcium (mg/dl) & $10,17 \pm 0,8$ & $10,05 \pm 1,2$ & $10,24 \pm 0,9$ & 0,21 & 0,813 \\
\hline Phosphore (mg/dl) & $3,33 \pm 0,7$ & $3,94 \pm 0,6$ & $3,35 \pm 0,6$ & 5,67 & $0,005^{*}$ \\
\hline Glucose (mmol/l) & $4,71 \pm 0,7$ & $4,93 \pm 1,7$ & $5,48 \pm 1,34$ & 2,01 & 0,141 \\
\hline
\end{tabular}


Tableau 2 : Moyennes des paramètres biochimiques du haut niveau de compétition.

\begin{tabular}{|c|c|c|c|}
\hline \multirow{2}{*}{ Paramètres biochimiques } & \multicolumn{3}{|c|}{ Niveau haut $(n=5)$} \\
\hline & Période $\left(t_{1}\right)$ & Période $\left(t_{2}\right)$ & Période $\left(t_{3}\right)$ \\
\hline Hématocrite (\%) & $35,4 \pm 2,3^{c}$ & $50,2 \pm 3,1^{\mathrm{a}}$ & $37,8 \pm 1,4^{\mathrm{b}}$ \\
\hline CK (UI/l) & $178,4 \pm 27$ & $211 \pm 43,7$ & $182,8 \pm 21,5$ \\
\hline ASAT (UI/l) & $218,6 \pm 21,5$ & $251 \pm 25,8$ & $224,8 \pm 23,4$ \\
\hline LDH (UI/l) & $361,2 \pm 100,5$ & $369,8 \pm 68$ & $396,8 \pm 103,7$ \\
\hline Urée (mmol/l) & $6 \pm 0,8$ & $6,1 \pm 0,3$ & $6,7 \pm 0,4$ \\
\hline Créatinine $(\mu \mathrm{mol} / \mathrm{l})$ & $137,6 \pm 9,9$ & $153,4 \pm 9,6$ & $159,2 \pm 11,3$ \\
\hline Protéines totales $(\mathrm{g} / \mathrm{l})$ & $55,18 \pm 5,7^{\mathrm{c}}$ & $63,9 \pm 10,3^{\mathrm{a}}$ & $55,16 \pm 6,5^{\mathrm{b}}$ \\
\hline Albumine $(\mathrm{g} / \mathrm{l})$ & $25,72 \pm 1,5^{\mathrm{c}}$ & $26,64 \pm 1,7^{\mathrm{a}}$ & $26,62 \pm 2,5^{\mathrm{b}}$ \\
\hline Magnésium (mg/dl) & $2,11 \pm 0,3^{\mathrm{a}}$ & $2,01 \pm 0,2^{\mathrm{b}}$ & $1,73 \pm 0,3^{\mathrm{c}}$ \\
\hline Calcium (mg/dl) & $9,52 \pm 0,3^{\mathrm{a}}$ & $8,87 \pm 0,2^{\mathrm{c}}$ & $9,41 \pm 0,8^{\mathrm{b}}$ \\
\hline Phosphore (mg/dl) & $3,98 \pm 0,4^{\mathrm{b}}$ & $4,69 \pm 0,4^{\mathrm{a}}$ & $3,78 \pm 0,5^{\mathrm{c}}$ \\
\hline Glucose (mmol/l) & $4,7 \pm 0,4^{\mathrm{c}}$ & $7,17 \pm 1,4^{\mathrm{a}}$ & $6,42 \pm 1^{\mathrm{b}}$ \\
\hline
\end{tabular}

Les moyennes des paramètres en ligne affectées de lettres $(\mathrm{a}, \mathrm{b}, \mathrm{c})$ différentes sont significativement différentes $(\mathrm{p}<0,05)$.

Tableau 3 : Moyennes des paramètres biochimiques du niveau moyen de compétition.

\begin{tabular}{|c|c|c|c|}
\hline \multirow{2}{*}{ Paramètres biochimiques } & \multicolumn{3}{|c|}{ Niveau moyen $(n=6)$} \\
\hline & Période $\left(t_{1}\right)$ & Période $\left(t_{2}\right)$ & Période $\left(t_{3}\right)$ \\
\hline Hématocrite (\%) & $35,33 \pm 2,8$ & $49,66 \pm 3,1$ & $36,66 \pm 2,5$ \\
\hline CK (UI/l) & $163,66 \pm 26,5$ & $184,83 \pm 32,5$ & $176,66 \pm 56,8$ \\
\hline ASAT (UI/l) & $235 \pm 51,3$ & $255,16 \pm 54,3$ & $220,5 \pm 56,9$ \\
\hline LDH (UI/l) & $465,3 \pm 191,4$ & $534,6 \pm 177$ & $571,3 \pm 186,7$ \\
\hline Urée (mmol/l) & $5,08 \pm 1,3$ & $5,42 \pm 1,1$ & $6,04 \pm 1,2$ \\
\hline Créatinine $(\mu \mathrm{mol} / \mathrm{l})$ & $132,83 \pm 17,4$ & $148,5 \pm 20,7$ & $142,16 \pm 19$ \\
\hline Protéines totales $(\mathrm{g} / \mathrm{l})$ & $58,51 \pm 4,4$ & $64,21 \pm 2,6$ & $58,33 \pm 2,3^{\mathrm{b}}$ \\
\hline Albumine $(\mathrm{g} / \mathrm{l})$ & $24,66 \pm 3,9$ & $27,26 \pm 3,1^{\mathrm{b}}$ & $25,01 \pm 3,7$ \\
\hline Magnésium (mg/dl) & $3,1 \pm 0,4$ & $2,92 \pm 0,2^{\mathrm{b}}$ & $2,77 \pm 0,5^{\mathrm{b}}$ \\
\hline Calcium (mg/dl) & $10,05 \pm 0,6$ & $10,43 \pm 1,4^{\mathrm{b}}$ & $10,02 \pm 0,8^{b}$ \\
\hline Phosphore (mg/dl) & $3,04 \pm 0,7$ & $3,5 \pm 0,5^{\mathrm{b}}$ & $3,27 \pm 0,7$ \\
\hline Glucose (mmol/l) & $4,19 \pm 0,2$ & $3,91 \pm 0,6^{\mathrm{b}}$ & $4,30 \pm 0,1^{\mathrm{b}}$ \\
\hline
\end{tabular}

Tableau 4 : Moyennes des paramètres biochimiques du niveau débutant de compétition.

\begin{tabular}{lccc}
\hline \multirow{2}{*}{$\begin{array}{l}\text { Paramètres } \\
\text { biochimiques }\end{array}$} & \multicolumn{3}{c}{ Niveau débutant $(\mathbf{n}=\mathbf{1 2})$} \\
\cline { 2 - 4 } & Période $\left(\mathbf{t}_{\mathbf{1}}\right)$ & Période $\left(\mathbf{t}_{\mathbf{2}}\right)$ & Période $\left(\mathbf{t}_{\mathbf{3}}\right)$ \\
\hline Hématocrite $(\%)$ & $35,41 \pm 3,8$ & $48,75 \pm 3,1$ & $37 \pm 1,9$ \\
CK (UI/l) & $181,33 \pm 53,1$ & $233,58 \pm 82$ & $196,33 \pm 60,9$ \\
ASAT (UI/l) & $217,16 \pm 38,5$ & $246,91 \pm 41,2$ & $228,25 \pm 44,1$ \\
LDH (UI/l) & $412,1 \pm 135,3$ & $478,7 \pm 110,9$ & $465,1 \pm 127,8$ \\
\hline
\end{tabular}




\begin{tabular}{|c|c|c|c|}
\hline Urée (mmol/l) & $6,06 \pm 1,1$ & $6,22 \pm 0,9$ & $6,67 \pm 0,9$ \\
\hline Créatinine ( $\mu \mathrm{mol} / \mathrm{l})$ & $138,25 \pm 11,6$ & $164,5 \pm 18,7$ & $156,83 \pm 14,3$ \\
\hline Protéines totales $(\mathrm{g} / \mathrm{l})$ & $63,7 \pm 5,1^{\mathrm{c}}$ & $68,89 \pm 6,2^{\mathrm{a}}$ & $63,9 \pm 6,3^{\mathrm{b}}$ \\
\hline Albumine $(\mathrm{g} / \mathrm{l})$ & $28,5 \pm 2,1^{\mathrm{b}}$ & $30,74 \pm 1,5^{\mathrm{a}}$ & $28,05 \pm 3,7^{\mathrm{c}}$ \\
\hline Magnésium (mg/dl) & $2,51 \pm 0,4^{\mathrm{c}}$ & $2,78 \pm 0,4^{\mathrm{a}}$ & $2,57 \pm 0,5^{b}$ \\
\hline Calcium (mg/dl) & $10,5 \pm 0,9^{b}$ & $10,36 \pm 1^{\mathrm{c}}$ & $10,7 \pm 0,7^{\mathrm{a}}$ \\
\hline Phosphore (mg/dl) & $3,2 \pm 0,6^{\mathrm{c}}$ & $3,84 \pm 0,6^{\mathrm{a}}$ & $3,22 \pm 0,6^{\mathrm{b}}$ \\
\hline Glucose (mmol/l) & $4,97 \pm 0,8^{\mathrm{b}}$ & $4,51 \pm 1,4^{\mathrm{c}}$ & $5,68 \pm 1,4^{\mathrm{a}}$ \\
\hline
\end{tabular}

Tableau 5 : Moyennes des paramètres biochimiques des chevaux en compétition régulière.

\begin{tabular}{lccc}
\hline Paramètres & \multicolumn{3}{c}{ Chevaux $($ Compétition régulière) $(\mathbf{n = 6})$} \\
\cline { 2 - 4 } biochimiques & Période $\left(\mathbf{t}_{\mathbf{1}}\right)$ & ${\text { Période }\left(\mathbf{t}_{\mathbf{2}}\right)}$ & ${\text { Période }\left(\mathbf{t}_{\mathbf{3}}\right)}$ \\
Hématocrite $(\%)$ & $35,05 \pm 2,2$ & $49,35 \pm 3,2$ & $36,88 \pm 1,9$ \\
CK $(\mathrm{UI} / \mathrm{l})$ & $174,64 \pm 38$ & $215,94 \pm 70,1$ & $186,05 \pm 48,8$ \\
ASAT $(\mathrm{UI} / \mathrm{l})$ & $215,94 \pm 40,6^{\mathrm{b}}$ & $242,64 \pm 43,5^{\mathrm{a}}$ & $215,41 \pm 42,5^{\mathrm{c}}$ \\
LDH $(\mathrm{UI} / \mathrm{l})$ & $381,52 \pm 148,2$ & $458,58 \pm 141,4$ & $473,41 \pm 153,8$ \\
Urée $(\mathrm{mmol} / \mathrm{l})$ & $6,14 \pm 0,9$ & $5,99 \pm 0,9$ & $6,61 \pm 0,9$ \\
Créatinine $(\mu \mathrm{mol} / \mathrm{l})$ & $136,52 \pm 13,9^{\mathrm{c}}$ & $152,52 \pm 16,5^{\mathrm{a}}$ & $151,05 \pm 15,8^{\mathrm{b}}$ \\
Protéines totales $(\mathrm{g} / \mathrm{l})$ & $59,15 \pm 6,1^{\mathrm{c}}$ & $65,72 \pm 7,3$ & $59,52 \pm 7^{\mathrm{b}}$ \\
Albumine $(\mathrm{g} / \mathrm{l})$ & $25,99 \pm 3^{\mathrm{c}}$ & $28,21 \pm 2,8^{\mathrm{a}}$ & $26,24 \pm 3,5^{\mathrm{b}}$ \\
Magnésium $(\mathrm{mg} / \mathrm{dl})$ & $2,69 \pm 0,5$ & $2,67 \pm 0,5$ & $2,47 \pm 0,6$ \\
Calcium $(\mathrm{mg} / \mathrm{dl})$ & $10,23 \pm 0,9$ & $10,17 \pm 1,3$ & $10,24 \pm 1$ \\
Phosphore $(\mathrm{mg} / \mathrm{dl})$ & $3,38 \pm 0,7$ & $3,94 \pm 0,6$ & $3,44 \pm 0,6$ \\
Glucose $(\mathrm{mmol} / \mathrm{l})$ & $4,47 \pm 0,5^{\mathrm{c}}$ & $4,81 \pm 1,8^{\mathrm{b}}$ & $5,05 \pm 1,1^{\mathrm{a}}$ \\
\hline Les moyennes des paramètres en ligne affectées de lettres (a,b,c) différentes sont significativement différentes $(\mathrm{p}<0,05)$
\end{tabular}

Tableau 6 : Moyennes des paramètres biochimiques des chevaux n'ayant pas de compétition.

\begin{tabular}{lccc}
\hline Paramètres & \multicolumn{3}{c}{ Chevaux $($ Pas encore de compétition) $(\mathbf{n}=\mathbf{1 7})$} \\
\cline { 2 - 4 } biochimiques & Période $\left(\mathbf{t}_{\mathbf{1}}\right)$ & Période $\left(\mathbf{t}_{\mathbf{2}}\right)$ & Période $^{\left(\mathbf{t}_{\mathbf{3}}\right)}$ \\
Hématocrite $(\%)$ & $36,33 \pm 5,2$ & $49,16 \pm 2,5$ & $37,66 \pm 2,3$ \\
CK $(\mathrm{UI} / \mathrm{l})$ & $180,16 \pm 55,6$ & $216 \pm 59,8$ & $194,5 \pm 66,5$ \\
ASAT $(\mathrm{UI} / \mathrm{l})$ & $239,66 \pm 27,6^{\mathrm{c}}$ & $270,66 \pm 22,7^{\mathrm{a}}$ & $254 \pm 29,8^{\mathrm{b}}$ \\
LDH $(\mathrm{UI} / \mathrm{l})$ & $509,66 \pm 78,3$ & $501 \pm 108,5$ & $491 \pm 144,3$ \\
Urée $(\mathrm{mmol} / \mathrm{l})$ & $5,51 \pm 1,2$ & $6 \pm 1$ & $6,23 \pm 0,9$ \\
Créatinine $(\mu \mathrm{mol} / \mathrm{l})$ & $137,16 \pm 9,4$ & $173,16 \pm 15,9^{\mathrm{b}}$ & $160,5 \pm 15,8$ \\
Protéines totales & $64,31 \pm 4,2$ & $69,03 \pm 4,9$ & $63,45 \pm 4,2$ \\
(g/l) & & & \\
Albumine $(\mathrm{g} / \mathrm{l})$ & $29,46 \pm 1,3^{\mathrm{b}}$ & $31 \pm 1^{\mathrm{a}}$ & $28,96 \pm 3,3^{\mathrm{c}}$ \\
Magnésium $(\mathrm{mg} / \mathrm{dl})$ & $2,26 \pm 0,4$ & $2,60 \pm 0,5$ & $2,33 \pm 0,5$ \\
Calcium $(\mathrm{mg} / \mathrm{dl})$ & $10,01 \pm 0,3$ & $9,72 \pm 0,3$ & $10,26 \pm 0,5$ \\
Phosphore $(\mathrm{mg} / \mathrm{dl})$ & $3,18 \pm 0,7$ & $3,91 \pm 0,8$ & $3,10 \pm 0,7$ \\
Glucose $(\mathrm{mmol} / \mathrm{l})$ & $5,39 \pm 0,7^{\mathrm{b}}$ & $5,3 \pm 1,5^{\mathrm{c}}$ & $6,70 \pm 1,1^{\mathrm{a}}$ \\
\hline \multicolumn{2}{c}{ Les moyennes des paramètres en ligne affectées de lettres $(\mathrm{a}, \mathrm{b}, \mathrm{c})$ différentes sont significativement différentes } \\
\multicolumn{2}{c}{$(\mathrm{p}<0,05)}$.
\end{tabular}




\section{DISCUSSION}

L'hématocrite et les protéines totales ont varié significativement en fonction de la période de prélèvement. La valeur élevée de l'hématocrite après l'effort (période $\mathrm{t}_{2}$ ) pourrait s'expliquer par des changements importants du volume plasmatique à la suite de la déshydratation ou de la libération de globules rouges par la rate avec l'exercice. Pendant l'effort, le cheval possède la capacité d'augmenter son hématocrite de façon spectaculaire par la contraction de sa rate (Piccione et al., 2007).

L'augmentation significative de la concentration des protéines totales après l'effort (période $\mathrm{t}_{2}$ ) pourrait être due à la déshydratation. Les fortes concentrations des protéines totales et de l'albumine indiquent le degré de déshydratation chez le cheval après l'exercice (Kedzierski et al., 2009). L'augmentation des protéines plasmatiques après l'exercice s'expliquerait par les flux de protéines et une baisse du volume du liquide intravasculaire due à la transpiration. Cependant, Lafon (2008) a observé sur un cheval bien préparé une hémodilution avec baisse des protéines totales et de l'albumine.

De même, les activités des enzymes musculaires (l'ASAT et la CK) étaient plus élevées après l'effort (période $t_{2}$ ) comparés aux deux autres périodes $t_{1}$ et $t_{3}$. Ceci pourrait être dû à une hausse de la perméabilité membranaire. En effet, si l'activité musculaire est importante, cela entraîne une augmentation de la perméabilité membranaire donc, une élévation des enzymes musculaires (valberg, 2009). Ces résultats corroborent ceux d'études antérieures chez les chevaux de sport (Çötelioğlu et al., 2001 ; Sribhen et al., 2007 ; Vincze et al., 2012; Hura et al., 2013).

Par ailleurs, les valeurs de la créatinine ont varié significativement en fonction de la période de prélèvement. La valeur plus élevée de la créatinine des chevaux après l'effort (période $\mathrm{t}_{2}$ ) pourrait s'expliquer par l'exercice musculaire. Il a été démontré que la concentration de la créatinine augmente avec l'exercice (Nogueira et al., 2002 ; Seppa et al.,
2009). Cette augmentation de la créatininémie est confirmée par l'élévation des enzymes musculaires déjà mentionnée. En effet, la créatininémie est beaucoup plus élevée chez les chevaux athlètes que chez les chevaux sédentaires (Piccione et al., 2009).

L'augmentation significative de la concentration du phosphore après l'effort (période $\mathrm{t}_{2}$ ) pourrait être due à une importante destruction de l'adénosine triphosphate (ATP) durant l'exercice (Santos et al., 2001). La concentration du phosphore significativement plus élevée chez les chevaux de haut niveau corrobore les variations observées après l'effort (période $\mathrm{t}_{2}$ ) en fonction des périodes de prélèvement. D'autres auteurs soutiennent que l'augmentation de la concentration sérique du phosphore durant l'exercice résulterait probablement d'une hémoconcentration induite par l'exercice (Inoue et al., 2002). Cependant, Arslan et al. (2002) ont montré que l'augmentation du niveau de phosphore plasmatique durant et à la fin de l'exercice n'était pas significative chez les chevaux de course anglais.

De même, l'augmentation significative du glucose aux périodes $t_{2}$ et $t_{3}$ des chevaux de haut niveau pourrait s'expliquer par l'effet du stress ou par le fait que pendant l'effort, le muscle utilise davantage ses importantes réserves de glycogène que le glucose circulant. Dans des études antérieures, il a été rapporté que l'exercice intense augmente les transporteurs de glucose insulino-sensible, qui jouent le rôle de médiateur d'absorption du glucose dans le muscle squelettique (Tomás et al., 2002; Asplin et al., 2010). L'exercice augmente le taux d'utilisation du glucose en contractant les muscles squelettiques (Poortmans et Boisseau, 2004). En outre, Cornus (2010) a montré une augmentation de la glycémie chez un jeune cheval qui a présenté un stress lors de la contention. Le rythme des entraînements et de compétitions imposé aux chevaux de haut niveau peut même provoquer des pathologies liées au stress (Frank et al., 2010). 
Toutefois, le taux de glucose après l'effort $\left(\mathrm{t}_{3}\right)$ était significativement inférieur chez les chevaux qui compétissaient régulièrement que chez les chevaux qui n'avaient pas encore effectué de compétition officielle. Cela supposerait que les chevaux de haut niveau contrôlent mieux leur état de stress que les moins bien entraînés. Ainsi, pour optimiser les performances individuelles, les chevaux de sport doivent être en parfaite condition physique et avoir un bon état psychologique (McBride et Mills, 2012). Cette théorie a été confirmée par d'autres auteurs qui ont constaté que la concentration $\mathrm{du}$ glucose après la course était significativement plus basse chez les chevaux de bonne performance que les chevaux de faible performance. (Adamu et al., 2010 ; 2013).

Le niveau de compétition a justifié certaines variations significatives des paramètres biochimiques. Ainsi, les concentrations du calcium et du magnésium aux périodes $t_{2}$ et $t_{3}$ étaient plus basses chez les chevaux de haut niveau en raison des pertes dues à la sudation importante. En effet, si l'activité musculaire est importante, le calcium et le magnésium sont perdus en grande quantité dans la sueur en raison de la déshydratation. L'exercice intense augmente apparemment les pertes de magnésium par voies urinaires et par la sueur qui peuvent accroître les besoins en magnésium de 10 à $20 \%$ (Nielsen et al., 2006). Ces résultats sont similaires à ceux d'Inoue et al. (2002) qui ont rapporté que la perte de calcium par sudation était non négligeable chez les chevaux pendant l'exercice.

La fréquence de compétition a eu un effet significatif sur les valeurs de l'ASAT, de la créatinine, de l'albumine et du glucose. La valeur de l'ASAT significativement plus basse chez les chevaux qui participaient régulièrement à des compétitions pourrait être due à l'entraînement. En effet, il a été rapporté que l'entraînement occasionne une diminution des valeurs des enzymes musculaires (Çötelioğlu et al., 2001; Padalino et al., 2007 ;
Lafon, 2008). La créatininémie plus basse chez les chevaux bien entraînés est confirmée par la diminution de l'ASAT observée chez ces mêmes chevaux. Les chevaux très performants ont une créatininémie plus basse que les chevaux de faible performance (Adamu et al., 2013).

\section{Conclusion}

Cette étude a permis d'évaluer les modifications des paramètres biochimiques des chevaux de courses de saut d'obstacle du Sénégal avant et après l'effort. Ces données pourraient être d'une grande utilité pour le vétérinaire équin dans l'appréciation de la condition physique et de la performance des chevaux de course. La finalité de ce travail est d'offrir aux entraîneurs et aux propriétaires, par le biais de leur médecin vétérinaire, des méthodes de gestion qui permettront l'optimisation et la pérennité des performances de leurs chevaux.

\section{CONFLIT D'INTERET}

Les auteurs déclarent qu'il n'existe aucun conflit d'intérêt.

\section{CONTRIBUTIONS DES AUTEURS}

$\mathrm{AS}, \mathrm{AB}$ et GJS ont rédigé le protocole expérimental, $\mathrm{AB}, \mathrm{MK}$ et RST ont effectué la mise en œuvre de l'expérience de terrain. AB, MK et AS ont réalisé les analyses de laboratoire ainsi que leur interprétation. Tous les auteurs ont contribué à la rédaction du manuscrit.

\section{REMERCIEMENTS}

Nous remercions le Directeur Général de l'EISMV pour nous avoir facilité les conditions de travail. Nous sommes très reconnaissants à l'endroit de Mlle Camille EGUE pour son aide pour les analyses biochimiques. Nous exprimons aussi notre gratitude à tous les gendarmes du service vétérinaire et à tous ceux de l'Escadron Monté du Camp Diéry Diallo de Dakar pour leur aide lors de la mise en œuvre du protocole et la réalisation des prélèvements. 


\section{REFERENCES}

Adamu L, Adzahan NM, Abdullah R, Ahmad B. 2010. Effects of race distance on physical, hematological and biochemical parameters of endurance horses. Am. $J$. Anim. Vet. Sci., 5(4): 244-248. DOI :10.3844/ajavsp.2010.244.248.

Adamu L, Noraniza M A, Rasedee A, Bashir A. 2013. Effect of age and performance on physical, hematological, and biochemical parameters in endurance horses. J. Eq. Vet. Sci., 33(6): 415-420. DOI : http://dx.doi.org/10.1016/j.jevs. 2012.07.015.

Arslan M, Ozcan M, TosunCotelioglu U, Matur E. 2002. The effects of physical exercise on some plasma enzymes and $\mathrm{Ca}$ and $\mathrm{P}$ levels in race horses. J. Fac. Vet. Med. Univ. Istanbul., 28: 91-97. (http://vetjournal.istanbul.edu.tr/archive/2 002-1/91_97.pdf).

Art T, Amory H, Lekeux P. 2000. Notions de base de physiologie de l'effort. Prat. Vét. Eq., 32: 247-254. DOI :http://hdl.handle.net/2268/8290.

Asplin KE, Curlewis JD, McGowan CM, Pollitt CC, Sillence MN. 2010. Glucose transport in the equine hoof. Eq. Vet J., 43: 196-201. DOI : $10.1111 /$ j.20423306.2010.00127.x.

Bathily A, Sow A, Kalandi M, Sawadogo GJ. 2014. Paramètres morphobiométriques et biochimiques des chevaux de traction au Sénégal. Int. J. Biol. Chem. Sci., 8(6): 2731-2739.

DOI http://dx.doi.org/10.4314/ijbcs.v8i6.32.

Cornus J. 2010. Valeurs usuelles en biochimie sérique chez le cheval selle Français: Données du laboratoire biochimique de l'ENVA Thèse : Méd. Vét, Alfort, p. 90.

Çötelioğlu Ü, Arslan M, Matur E, Bakirel U, Özcan M, Tosun C. 2001. The effects of physical exercice on some physiological parameters, plasma CK and LDH levels in horses that are breeded as race horses. J. Fac. Vet. Med. Univ. Istanbul., 27(2): 609-615. (http://vetjournal.istanbul.edu.tr/archive/2 001-2/609_615.pdf).

Frank N, Geor RJ, Bailey SR., Durham A.E., Johnson P.J. 2010.Equine metabolic syndrome. J. Vet. Internal Med., 24(3): 467-475. DOI : $10.1111 /$ j.19391676.2010.0503.x.

Hura V, Novotný F, Boldižár M, Rédl M, Noskovičová J, Horňák S, Kováč G. 2013. Biochemical responses to a nonstandard exercise in horses trained for jumping. Acta Vet.Brno., 82(2): 161-167. DOI : $10.2754 / \mathrm{avb201382020161.}$

Inoue $\mathrm{Y}$, Osawa T, Matsui A, Asai Y, Murakami Y, Matsui T, Yano H. 2002. Changes of Serum Mineral Concentrations in Horses during Exercise.Asian-Aust. J. Anim. Sci., 15(4): 531-536. DOI : http://dx.doi.org/10.5713/ ajas.2002.531.

Kedzierski W, Bergero D, Assenza A. 2009. Trends Of Hematological And Biochemical Values In The Blood Of Young Race Horses During Standardized Field Exercise Tests. Acta Vet. Belgrad, 59(5-6) : 457-466. DOI : 10.2298/AVB0906457.

Lafon M. 2008. Sciences \& pratique. Myopathie atypique : de nouveau des cas en 2008. La Dépêche Vétérinaire $N^{\circ} 976$ du 16 au 22 février.

Martin-Rosset W. 1990. L'Alimentation des Chevaux: Techniques et Pratiques. Institut National de la Recherche Agronomique (INRA). Paris, France.

Mcbride SD, Mills DS. 2012. Psychologicalfactorsaffectingequine performance. Vet. Res., 8: 180. DOI: 10.1186/1746-6148-8-180.

Nielsen FH, Lukaski HC. 2006. Update on the relationship between magnesium and exercise. Magnesium Res., 19(3): 180189. DOI :10.1684/mrh.2006.0060.

Nogueira NP, Barnabe RC, Bedran-De-Castro JC, Moreira AF, Fernandes WR, Mirandola RMS, Howard DL. 2002. Serum cortisol, lactate and creatinine concentrations in Thoroughbred fillies of 
different ages and states of training. Braz. J. Vet. Res. Anim. Sci., 39(1):54-57. DOI : http://dx.doi.org/10.1590/S141395962002000100010.

Padalino B, Rubino G, Centoducati P, Petazzi F. 2007. Training versus Overtraining: Evaluation of Two Protocols. J. Eq. Vet. Sci., 27: 28-31. DOI: http://dx.doi.org/10.1016/j.jevs.2006.11.0 13.

Piccione C, Giannetto C, Fazio F, Di Mauro F, Caola G. 2007. Haematological response to different workload in jumper horses. Bulgarian J. Vet. Med., 10(4): 2128. (http://tru.uni-sz.bg/bjvm/bjvm.htm)

Poortmans JR, Boisseau N. 2003. Les mécanismes biochimiques de la contraction musculaire: la typologie des fibres. In Biochimie des Activités Physiques. De Boeck Université: Bruxelles; 45-82.

Santos SA, Silva RAMS, AzevedoJRM, Mello MAR, SoaresAC, SibuyaCY, AnarumaCA. 2001. Serum electrolyte and total protein alterations in Pantaneiro horse during long distance exercise. Arq.Bras. Med. Vet. Zootech., 53(3): 351357. DOI : http://dx.doi.org/10.1590/ S0102-09352001000300013

Seppa GS, Hess TM, Kowal RJ, Santos OJ. 2009. Comparison of plasma biochemical parameters of exhausted and non- exhausted horses participating in 1000 to $2000 \mathrm{~m}$ races. J. Eq. Vet. Sci., 29(5): 302303. DOI: http://dx.doi.org/10.1016/ j.jevs.2009.04.008.

Sribhen C, Sitthichaiyakul A, Kongpiromchean Y, Sribhen K. 2007. Influence of Training Exercise on Clinical Plasma Chemistry Parameters and Cardiac Markers in Race Horses. Kasetsart J. Nat. Sci., 41(3): 478-483. (http://rdi.ku.ac.th/KU_Journal/Sciences/ doc/KU41\%283\%29.pdf).

Tomás E, Zorzano A, Ruderman NB. 2002. Exercise and insulin signaling: a historical perspective. J. Appl. Physiol., 93: 765-772. DOI : 10.1152/japplphysiol.00267.2002.

Valberg SJ. 2009. The musculoskeletal system. Heritable muscle disease. In Current Therapy in Equine Medicine, Robinson NE, Sprayberry KA (eds). Saunders, Elsevier: St. Louis, USA; 461468.

Vincze A, Szabó C, Hevesi Á, Veres S, Ütő D. 2012. The effect of workload type and baseline covariate on the response of plasma biochemical parameters in show jumpers. Acta Argri. Sloven. Suppl., 3: 317-321. (http://aas.bf.unilj.si/zootehnika/supl/3-2012/PDF/3-2012317-321.pdf). 